\title{
OBSERVACIONES SOBRE EL PRINCIPIO PRECAUTORIO EN ARGENTINA
}

\author{
VALERIA BERROS \\ Docente Investigadora del Centro de Investigaciones en Derecho \\ Becaria Postdoctoral del CONICET \\ Facultad de Ciencias Jurídicas y Sociales de la UNL (Argentina) \\ vberros@fcjs.unl.edu.ar
}

Recibido: 30 de septiembre de 2013 / Aceptado: 28 de noviembre de 2013

RESUMEN: El objetivo del presente trabajo consiste en presentar una serie de observaciones sobre la aplicación del principio precautorio en Argentina. Con ese fin se selecciona como caso de estudio el problema del uso de agrotóxicos en este país, que es analizado a partir de dos ejes. El primero hace referencia a las decisiones judiciales sobre el tema que han proliferado en los últimos años. El segundo indaga en las estructuras y procedimientos que desde el Estado intentan gestionar este problema, a los efectos de dilucidar los desafíos en el ámbito del diseño institucional sobre el gobierno de riesgos controvertidos.

RESUM: L'objectiu del present treball consisteix en presentar una sèrie d'observacions sobre l'aplicació del principi precautori a Argentina. Amb aquesta finalitat es selecciona com a cas d'estudi el problema de l'ús d'agrotòxics en aquest país, que s'analitza partint de dos eixos. El primer fa referència a les decisions judicials sobre el tema, que han proliferat els darrers anys. El segon, indaga a les estructures i procediments que, des de l'Estat, intenten gestionar aquest problema, als efectes d'escatir els desafiaments en l'àmbit del disseny institucional, sobre el govern dels riscos controvertits.

ABSTRACT: The aim of the present work consists in presenting a series of observations on the application of the precautionary principle in Argentina. With this purpose we select the case of the use of pesticides in this country which is studied from two axes. The first one is the judicial decisions on the topic that have proliferated in the last years. The second 
one is the structures and procedures that try to manage this problem. The aim in this second part is to elucidate the challenges for the area of the institutional design for the government of controversial risks.

PALABRAS CLAVE: Principio Precautorio - Riesgos controvertidos - Agrotóxicos - Argentina.

PARAULES CLAU: Principi precautori - Riscos controvertits - Agrotòxics Argentina.

KEY WORDS: Precautionary Principle — Controversial Risks — Pesticides — Argentina.

SUMARIO: I. Introducción. II. Presentación de los principales planteamientos judiciales. 1. El problema de la distancia de las fumigaciones ante la jurisdicción. 2. La discusión sobre la constitucionalidad de normas locales que prohíben o limitan el uso de agrotóxicos. III. La construcción de espacios y mecanismos para la decisión sobre riesgos. 1. Diseños institucionales para la evaluación de riesgos. 2. Procedimientos legales referidos a riesgos. IV. Algunas reflexiones finales. V. Referencias bibliográficas.

\section{INTRODUCCIÓN}

Actualmente, el principio precautorio se ubica como una herramienta para la decisión sobre riesgos controvertidos de ineludible relevancia en la denominada "sociedad del riesgo". El conjunto de reflexiones desarrolladas sobre este tema no han sido ajenas al campo del derecho. Por el contrario, en este último residen distintas problemáticas que colocan en un

\footnotetext{
${ }^{1}$ En 1986 se publica la obra "La sociedad del riesgo. Hacia una nueva modernidad" del sociólogo alemán Ulrich Beck que plantea el tránsito hacia una segunda modernidad en la que el conflicto ya no se centraría tanto en la distribución de la riqueza - característico de la primera modernidad -, como en la distribución de riesgos. BECK, U. La sociedad del riesgo. Hacia una nueva modernidad. Paidos. Barcelona. 1998. Sobre las diferentes teorías que se han elaborado sobre el problema del riesgo puede consultarse: LUPTON, D. Il rischio. Percezione, simboli, cultura Il Mulino, Universale Peperbacks. Bologna. 2003.

${ }^{2}$ Una construcción respecto de los desafíos que plantea la sociedad del riesgo al interior del campo jurídico en: HERMITTE, M. A. "La fondation juridique d'une société des sciences et des techniques par les crises et les risques", Burton-Jeangros, C ; Grosse C. y November, V. (dir.) Face au risque, Georg-L'Equinoxe, 2007 ; HERMITTE, M. A. "Los fundamentos jurídicos de la sociedad del riesgo. Un análisis de Ulrich Beck" Sozzo, G. (dir) El gobierno del riesgo Ediciones UNL. Santa Fe. 2007. La gestión de los riesgos científicotecnológicos también ha sido objeto de análisis desde el derecho en: ESTEVE PARDO, J. El desconcierto del
} 
primer plano a este principio y visibilizan importantes desafíos para el campo jurídico ${ }^{2}$. Caracterizado el mismo como una herramienta para la toma de decisiones en materia de ambiente y salud, se ha expandido su positivización normativa así como su aplicación en numerosas sentencias judiciales ${ }^{3}$. Fue así construyéndose una densa trayectoria, con diversas incorporaciones a normativas de distinto tenor, lo que incluye un número importante de normas soft law y de regulaciones a nivel internacional ${ }^{4}$, regional $^{5}$, nacional y aún local.

En esta trayectoria, se fueron planteando diferentes debates y desacuerdos, siendo considerado como un pilar del derecho ambiental ${ }^{6}$, como una "perspectiva" o "enfoque", o

\begin{abstract}
${ }^{2}$ Una construcción respecto de los desafíos que plantea la sociedad del riesgo al interior del campo jurídico en: HERMITTE, M. A. "La fondation juridique d'une société des sciences et des techniques par les crises et les risques", Burton-Jeangros, C ; Grosse C. y November, V. (dir.) Face au risque, Georg-L'Equinoxe, 2007 ; HERMITTE, M. A. "Los fundamentos jurídicos de la sociedad del riesgo. Un análisis de Ulrich Beck" Sozzo, G. (dir) El gobierno del riesgo Ediciones UNL. Santa Fe. 2007. La gestión de los riesgos científicotecnológicos también ha sido objeto de análisis desde el derecho en: ESTEVE PARDO, J. El desconcierto del Leviatán. Politica y derecho ante las incertidumbres de la ciencia, Marcial Pons. Madrid, 2009; ESTEVE PARDO, J. Técnica, riesgo y derecho. Tratamiento del riesgo tecnológico en el Derecho Ambiental, Ariel. Barcelona, 1999.
\end{abstract}

${ }^{3}$ Cabe señalar que, en primer término, este principio ha sido dispuesto para temas ambientales aunque luego se ha aplicado en temas de salud humana y de seguridad alimentaria, como testimonia la jurisprudencia del STJCE. NOIVILLE, C. Du bon gouvernement des risques. Le droit et la question du "risque acceptable ", Presses Universitaires de France, Paris, 2003; SOZZO, G. y BERROS, M. V. "Una agenda para el principio precautorio" Revista Crítica de Derecho Privado N 6 -2009, La Ley, Uruguay.

${ }^{4}$ En el ámbito internacional, la primera declaración que lo introduce es la Carta Mundial de la Naturaleza de 1982. En su primer estadio se relacionó con el problema de la polución marítima, verbigracia, en las Conferencias Internacionales de Protección del Mar del Norte (Bremen 1984, Londres 1987, Hague 1990, Esbjerg 1995). Más tarde se incorpora al campo de la contaminación atmosférica (Convención de Viena sobre la Protección de la Capa de Ozono 1985, Protocolo de Montreal sobre las Sustancias que agotan la Capa de Ozono 1987). Alrededor de una década después se lo introduce en un documento soft law de gran relevancia a nivel mundial: la Declaración de Rio de Janeiro sobre Medioambiente y Desarrollo de 1992, que lo recoge como Principio $\mathrm{N}^{\circ} 15$. El mismo año, además, se integra en la Convención de Cambio Climático y en la Convención de Diversidad Biológica. DE SADELEER, N. Environmental principles. From political slogans to legal rules, Oxford University Press. Oxford. 2002. Paralelamente, también se ha ido plasmando la referencia y/o aplicación del principio en el ámbito jurisdiccional internacional en la Corte Internacional de Justicia: Nuclear Tests (Nueva Zelanda v. Francia, 1995); Gabcikovo - Nagymaros (Hungría vs Eslovaquia, 1997); Aerial Herbicide Spraying (Ecuador v. Colombia, 2008); Pulp Mills on the River Uruguay (Argentina vs Uruguay, 2010) y en el Tribunal Internacional de Derecho del Mar: Nueva Zelanda c. Japón; Australia v. Japón (1999); Irlanda v. Reino Unido (2002) y Malasia v. Singapur (2003).

${ }^{5}$ A nivel regional la Unión Europea lo menciona como pilar de la política comunitaria en el Tratado de Maastricht de 1992 (art. 174.2). También se realizan referencias minoritarias o aplicaciones a nivel jurisprudencial en la Corte Europea sobre Derechos Humanos (CEDH), verbigracia en Balmer-Schafroth y otros vs Suiza (1997), y en el Tribunal Superior de Justicia (STJCE) como en el conocido asunto National Farmers Union, 1998. Asunto C-180/96.

${ }^{6}$ Se considera que el principio de precaución se torna un "principio fundador del derecho del medioambiente con el Informe Bruntland de 1988". HERMITTE, M. A. "La dissémination volontaire d'organismes 
bien, como una suerte de instrumento "anti-desarrollo científico tecnológico". Esto último forma parte de una discusión ya superada, dado que el principio de precaución, lejos de configurarse como anticientífico, permite focalizar la atención sobre problemáticas que requieren de una profundización del conocimiento ${ }^{7}$. Ahora bien, si se considera el cúmulo de producciones que en las últimas décadas integran tanto las aportaciones de la sociología del riesgo ${ }^{8}$, como aquellas que provienen de los estudios sociales de las ciencias - o social science studies $^{9}$-, el problema del principio precautorio no sólo podría enlazarse con el debate sobre su "anti-cientificidad". Los mencionados campos de estudio han tenido por objeto desvelar el funcionamiento del sistema científico y han arrojado luz sobre su carácter parlamentario $^{10}$. La ciencia es concebida hoy como un parlamento en cuyo interior existen diferentes posiciones que, también, son inestables en el tiempo. Así, se abre un interrogante sobre la racionalidad científica como único ámbito de producción de conocimiento. Asimismo, se postula la necesidad de pensar en términos de una "ecología de saberes"11

génétiquement modifiés dans l'environnement une première application du principe de précaution" Revue Juridique de l'Environnement $\mathrm{N}^{\circ} 3 / 93$.

7 NOIVILLE, C. "Science, décision, action: trois remarques à propos du principe de précaution" Petites affiches. 1er -2. N. 218-219, Paris, 2004.

${ }^{8}$ A la ya mencionada primera publicación de Ulrich Beck pueden agregarse, por ejemplo, del mismo autor: BECK, U. La sociedad del riesgo mundial, Paidos. Barcelona, 2007; BECK, U. La sociedad del riesgo global, Siglo XXI. Barcelona. 2002 así como la perspectiva de GIDDENS, A. (1993) Las consecuencias de la modernidad, Alianza. Madrid.1993 y, también, miradas aún más constructivistas como las de DOUGLAS, M. y WILDAVSKY, A. Risk and culture. An essay on the selection of technological and environmental dangers, University of California Press. California. 1983.

9 PESTRE, D. Introduction aux sciences studies, La Découverte. Paris. 2006.

${ }^{10}$ LATOUR, B. Politiques de la nature ¿Comment faire entrer les sciences en démocratie?, La Decouverte, Paris, 2004; LATOUR, B. Nous n'avons jamais été modernes. Essai d'anthropologie symétrique La Decouverte. Paris, 1997. Versión en español LATOUR, B. Nunca fuimos modernos. Ensayo de antropología simétrica Siglo XX Editores. Buenos Aires, 2007.

${ }^{11}$ SANTOS, B. Para descolonizar Occidente. Más allá del pensamiento abismal, CLACSO. Prometeo Libros. Buenos Aires, 2010. En esta obra se afirma que el paradigma científico moderno es revisable a la luz de otro paradigma emergente sobre las ciencias y, en ese marco, se construye la idea de ecología de saberes a manera de contra-epistemología. Se esgrime que ante la existencia de diferentes intervenciones en la realidad que pueden fundarse en distintos sistemas de conocimiento, la preferencia en relación a estos últimos debe ser otorgada a aquel que permita garantizar la mayor participación de los grupos sociales involucrados en el diseño, ejecución y control y en los beneficios de la intervención. La idea que subyace a la ecología de saberes es la de prudencia, en tanto aprendizaje de los otros conocimientos sin olvidar el propio. Se pone de relieve que en la generación de saberes no sólo se ubica a quienes forman parte del sistema científico institucionalizado sino, también, a quienes fueron localizados en la periferia, en el afuera, en lo no válido o cardinal que suele etiquetarse como conocimiento 'lego' o 'profano'. Sobre el conocimiento lego o profano y el desarrollo de ejercicios de epidemiología popular un texto fundacional publicado ya hace tres décadas: BROWN, P. "Epidemiology and Toxic Waste Contamination: Lay and Professional Ways of Knowing" Journal of Health and Social Behavior, Vol. 33, No. 3, 1992. 
que permitiría obtener una nueva imagen de la ciencia con otras maneras de producir conocimiento. Lo expuesto deja claro que existe un conjunto de reflexiones contemporáneas en torno a la producción de conocimiento que es imposible soslayar ante el principio de precaución y que colocan lo incierto en un espacio cardinal ${ }^{12}$.

En la actualidad, se otorga singular visibilidad a lo controvertido y a lo incierto y ello ha ganado espacio en distintas regulaciones. Ya se han efectuado algunas menciones sobre su recorrido en el ámbito internacional y regional. Esta emergencia y proliferación no constituye una excepción en el caso de América Latina en general y de Argentina en particular. En el Acuerdo Marco sobre Medioambiente del Mercado Común del Sur (MERCOSUR), se estipula que los Estados Parte "reafirman su compromiso con los principios enunciados en la Declaración de Río de Janeiro sobre Medio Ambiente y Desarrollo de 1992,"13. Entre dichos principios se encuentra el precautorio. En los diferentes países de América Latina que comenzaron por constitucionalizar el derecho al ambiente desde éticas antropocéntricas o más ligadas a los postulados del eco-centrismo, también el principio de precaución comienza a ser introducido. Y ello tiene su traslación en cuatro modalidades distintas: (i) incorporación a nivel constitucional ${ }^{14}$; (ii) incorporación y definición a nivel legal y, en algunos casos, con una referencia a sectores específicos sobre los cuales aplicar este principio $^{15}$; (iii) referencia sin conceptuación del principio $^{16}$; (iv) no adopción, al menos de modo expreso ${ }^{17}$.

12 Además de las anteriormente mencionadas obras sobre el problema del riesgo se podría agregar, verbigracia, CASTEL, R. La montée des incertitudes. Travail, protection, statut de l'individu, Editions du Seuil. Paris, 2009.

${ }^{13}$ En el Tratado constitutivo de la Unión de Naciones Suramericanas (UNASUR) no se efectúa una referencia explícita a los principios de política ambiental, aunque ésta se instituye como uno de los objetivos comunes de los Estados signatarios. En el caso del organismo intergubernamental, Comunidades de Estados Latinoamericanos y Caribeños (CELAC) en la reunión de diciembre 2011 se redacta un documento sobre desarrollo sostenible que remite a la Declaración de Río 1992.

${ }^{14}$ Constitución de Ecuador de 2008 en la que se hace referencia a la precaución tanto en relación a la naturaleza (Art. 73) como a la salud (Art. 32), el daño ambiental (Art. 397) y a sectores estratégicos, servicios y empresas públicas (Art. 313), lo cual es especialmente relevante dada la jerarquía que así le es atribuida al principio en el ordenamiento jurídico.

${ }^{15}$ Dentro de (ii) encontramos dos subgrupos. Uno que conceptualiza el principio y otro que, además, efectúa remisiones a problemáticas concretas respecto de los cuales posee especial valor. Dentro del segundo conjunto se destaca el caso de Colombia (Art. 11.inc 6 Ley N 99/1993) que, además, efectúa una remisión a los límites máximos de emisión, descarga o depósito de sustancias, productos o compuestos susceptibles de afectar el ambiente (Art. 5 .inc 25 Ley $\mathrm{N}^{\circ}$ 99/1993); la legislación (Art. 7 Ley $\mathrm{N}^{\circ}$ 28.611/2005) también efectúa una referencia especial a las radiaciones no ionizantes (Art. 116 Ley $\mathrm{N}^{\circ}$ 28.611/2005), Uruguay (Art. 6b. Ley $\mathrm{N}^{\circ} 17.283 / 2000$ ) que también lo dispone en el uso sostenible de la diversidad biológica (Art. 22 Ley 
Argentina se ubica en el segundo de los grupos mencionados. El principio precautorio se integra en el Ordenamiento Jurídico argentino a través de una norma nacional de “presupuestos mínimos"18: la Ley n² 25.675 de 2002 que fija la Política Ambiental Nacional, así como el repertorio de principios que la deben guiar en su artículo 4. Allí se estipula que: "Cuando haya peligro de daño grave o irreversible, la ausencia de información o certeza científica no deberá utilizarse como razón para postergar la adopción de medidas eficaces, en función de los costes, para impedir la degradación del medio ambiente". Dispuesto de ese modo desde 2002, es cierto que no ha inspirado tanto el diseño de espacios institucionales para la toma de decisiones, como sí estrategias judiciales. En este último sentido, el principio viene siendo utilizado por diferentes grupos que judicializaron conflictos y lo introdujeron entre sus argumentos, lo que también se verifica dentro de las líneas argumentales de jueces, juezas y tribunales que han decidido sobre conflictos de diversa índole. Lo expresado se ha manifestado en Argentina de manera evidente ante diferentes tipos de problemas, entre los que destacan la exposición a radiaciones no ionizantes o el uso de agrotóxicos ${ }^{19}$. Este último tema es el seleccionado como caso para ilustrar el análisis del presente trabajo. Por un lado, debido a la actualidad ineludible del tema en Argentina. Por el otro, considerando el incremento del número de acciones que intentan aplicar el principio de precaución para lograr que se prohíban fumigaciones o que se invaliden argumentos tendentes a la declaración de inconstitucionalidad de normas locales restrictivas sobre el uso de agrotóxicos. Un conjunto de decisiones serán objeto de

$\mathrm{N}^{\circ} 17.283 / 2000$ ). Luego se encuentran legislaciones en las que se positiviza la precaución y se determina su significado sin aunarlo en paralelo a cuestiones especiales: Venezuela (Art. 4.3 Ley N$^{\circ}$ 5833/2006) y República Dominicana (Art. 8 Ley N 64/2000).

${ }^{16}$ El Salvador (Art. 2.c Dec. Leg N²33/1998) y México (Art. 170 Lgeepa/1988 ref).

17 Aquí se encuentran Paraguay (que no posee una ley general dedicada al ambiente), Chile (Ley $\mathrm{N}^{\circ} 19.300 / 1994$ ), Bolivia (Ley N¹333/ 1992), Costa Rica (Ley $N^{\circ}$ 7554/1995), Cuba (Ley N 81/1997), Guatemala (Ley N 68/1986), Honduras (Ley N 104/1993), Nicaragua (Ley N² 217/1996), Panamá (Ley Nº 41/1998) y Brasil (Ley N6938/1981).

${ }^{18}$ El artículo $41 \mathrm{CN}$ incorporado en la reforma constitucional de 1994 afirma que la Nación es la encargada de dictar las leyes que contengan los presupuestos mínimos de protección en materia ambiental y las Provincias aquellas que fueran necesarias para complementarlas, sin que ello implique alterar las jurisdicciones locales.

${ }^{19}$ Sobre el tema específico de las radiaciones no ionizantes en Argentina: BERROS, M.V. "Principio precautorio como herramienta de gestión del riesgo ambiental" Revista de Derecho Ambiental $\mathrm{N}^{\circ} 13$, Lexis Nexis, 2008; BERROS, M. V. "La estandarización como tecnología precautoria globalizada. El caso de las radiaciones no ionizantes" Volumen 5, №2 Revista Prismas Direito, políticas públicas e mundializaçao. Colección Universitaria UNICEUB. Brasilia, 2008. Este tema también ha sido objeto de estudio en España: EMBID TELLO, A. Precaución y derecho. El caso de los campos electromagnéticos, Isutel. Madrid, 2010. 
estudio en la primera parte de este trabajo para pasar, luego, a analizar la necesidad de la existencia de espacios institucionales adecuados para la toma de decisiones en asuntos en que lo medular es lo controvertido o incierto. Estos ponen de relieve la necesidad de repensar los procedimientos y estructuras estatales adecuados para "situaciones de precaución" ${ }^{20}$. Situaciones que requieren la configuración de un proceso de apertura del debate sobre riesgos que, por su carácter colectivo, requieren de decisiones también construidas colectivamente.

\section{PRESENTACIÓN DE LOS PRINCIPALES PLANTEAMIENTOS JUDICIALES}

El problema del incremento del uso de agrotóxicos en Argentina está relacionado con la autorización del cultivo de semillas modificadas genéticamente $\mathrm{y}$, en particular, de soja modificada genéticamente tolerante al glifosato ${ }^{21}$. La referida autorización se realiza por medio de la Resolución n 167 del año 1996 de la Secretaría de Agricultura, Ganadería, Pesca y Alimentos de la Nación, que se configura como la primera de una serie de resoluciones de similar tenor. Lo expuesto, unido al aumento de los precios internacionales de los agroalimentos, generó una rápida expansión de lo que suele interpretarse en términos de 'proceso de sojización'. Este no sólo ha alterado el modo de cultivo en la región que tradicionalmente se destinó al trabajo agrario en el país sino que, además, ha generado consecuencias fuera de esa zona, extendiéndose sobre regiones boscosas o montes ${ }^{22}$. De forma paralela a esta expansión, se acrecentaron también las observaciones y denuncias sobre deforestación, degradación de los suelos, expansión del monocultivo, desarticulación de la agricultura familiar, desalojos de pequeños y medianos agricultores de sus tierras y

20 “...Una situación de precaución implica al menos: el productor del riesgo, el regulador, la víctima, pero asi también el experto, el periodista, que tienen a su cargo la tarea de medir, de evaluar los riesgos como de informar...”. EWALD, F. Le principe de précaution, Que sais je ? PUF. Paris, 2001, Pág. 70.

${ }^{21}$ El glifosato, N-(fosfonometil) glicina, es un herbicida creado en la década de los sesenta y utilizado para eliminar malezas indeseables en ambientes agrícolas, forestales y paisajísticos. Dentro del espectro de sustancias denominadas agroquímicos, encontramos los herbicidas (para la eliminación de malezas), los fungicidas (para combatir enfermedades causadas por hongos) y los insecticidas (destinados a combatir plagas)

${ }^{22}$ Informe "El avance de la frontera agropecuaria y sus consecuencias" de la Secretaría de Ambiente y Desarrollo Sustentable recuperado el 19 de octubre de 2011 de www.ambiente.gov.ar; Informes de la Red Agroforestal Chaco Argentina (REDAF) de 2009 y 2010 recuperados el 22 de noviembre de 2011 de http://redaf.org.ar/observatorio/. 
crecimiento de la periferia de grandes ciudades ${ }^{23}$. Y a ello se fueron sumando diferentes voces de alerta en relación a los efectos perjudiciales para la salud humana provocados por la exposición a agrotóxicos. En este último espacio se sumaron expertos de diferentes disciplinas científicas, así como profesionales de la salud y algunos ejercicios de epidemiología popular realizados por los propios afectados. Confluyen así miradas críticas y preocupadas por una serie de sintomatologías como las malformaciones congénitas, problemas respiratorios y de piel, cáncer, entre otros, que comenzaron a asociarse a la exposición a este tipo de sustancias.

En este contexto, y desde hace ya casi una década, diferentes escenarios de conflicto en relación al uso de agrotóxicos comenzaron a ser llevados a la instancia jurisdiccional. Con un objetivo meramente analítico se podrían agrupar del siguiente modo: (i) reclamaciones que ponen el acento en el incumplimiento de las distancias legalmente establecidas para la fumigación desde los ejidos urbanos, lo que se fija a través de leyes provinciales ${ }^{24}$; (ii) demandas por la declaración de inconstitucionalidad de normas locales que pretenden la prohibición o restricción del uso de agrotóxicos, presentadas por parte de empresas afectadas por disposiciones de tal naturaleza ${ }^{25}$.

\section{El problema de la distancia de las fumigaciones ante la jurisdicción}

El tema de la distancia-proximidad territorial respecto de la actividad de fumigaciones ya sea de modo aéreo o terrestre, es una de las reclamaciones con más trascendencia en este ámbito que se han planteado ante la justicia.

El caso Cavigliano Peralta, Viviana y otros c/ Municipalidad de San Jorge y otros s/ amparo resuelto por el Juzgado de Primera Instancia de Distrito en lo Civil, Comercial y Laboral de San Jorge y luego confirmado por la Sala II de la Cámara de Apelaciones en lo

\footnotetext{
${ }^{23}$ Informe "El avance...." (cit)

${ }^{24}$ Del conjunto de sentencias existentes se seleccionan aquí aquellas que poseen un rol relevante en cuanto al principio precautorio: Cavigliano Peralta, Viviana y otros c/ Municipalidad de San Jorge y otros s/ amparo, Sala II de la Cámara en lo Civil y Comercial de la ciudad de Santa Fe, 19.04.12 y aclaratoria de fecha 16.05.12; Monsalvo, Cristina y otros c/ Delaunay, Jorge s/ amparo, Corte Suprema de Justicia Provincia de Buenos Aires, 08.08.2012.

${ }^{25}$ Existen dos sentencias sobre este tema en materia de agrotóxicos: Speedagro SRL c/ Comuna de Arequito s/ medida cautelar, Sala II de la Cámara en lo Contencioso Administrativo de Rosario, 03.10.2011; Chañar Bonito S.A. c/ Municipio de Mendiolaza s/ amparo, Supremo Tribunal de Justicia de la Provincia de Córdoba, 18.09.2007.
} 
Civil y Comercial de Santa Fe, ha derivado en una de las sentencias más relevantes del último período en la que se ha realizado una aplicación del principio precautorio para fundamentar la prohibición de fumigar en una determinada zona.

La acción presentada es un amparo ${ }^{26}$, a través del cual, se solicita la prohibición de fumigaciones a ochocientos metros de uno de los barrios de la ciudad de San Jorge en la Provincia de Santa Fe. Se demanda a los propietarios del campo así como a los productores y al Estado local y provincial por omisión de control de los parámetros que rigen la materia conforme la legislación provincial vigente ${ }^{27}$.

El Tribunal a quo estima la acción aplicando el principio precautorio después de efectuar una revisión de los diferentes estudios que alertan sobre la toxicidad del glifosato, que es la sustancia que se utiliza en la zona cultivada. Sobre la base del principio de precaución, la segunda instancia confirma la decisión del Tribunal a quo y, asimismo, plantea que es necesario profundizar en la investigación sobre la toxicidad de la sustancia ${ }^{28}$. Para realizar esta tarea, no se recurre a los expertos de la lista oficial de peritos ${ }^{29}$, sino que se convoca a la Universidad Nacional del Litoral, sita en la localidad de Santa Fe, a fin de que informe sobre la toxicidad de la sustancia, en colaboración con el Ministerio de la Producción

\footnotetext{
${ }^{26}$ Art. 43: "Toda persona podrá interponer acción expedita y rápida de amparo, siempre que no exista otro medio judicial más idóneo, contra todo acto u omisión de autoridades públicas o de particulares, que en forma actual o inminente lesione, restrinja, altere o amenace con arbitrariedad o ilegalidad manifiesta, derechos y garantías reconocidos por esta Constitución, un tratado o una ley. En el caso, el juez podrá declarar la inconstitucionalidad de la norma en que se funda el acto u omisión lesiva. Podrán interponer esta acción contra cualquier forma de discriminación y en lo relativo a los derechos que protegen el ambiente, a la competencia, al usuario y al consumidor, así como a los derechos de incidencia colectiva en general, el afectado, el defensor del pueblo y las asociaciones que propendan a esos fines, registradas conforme a la ley, la que determinará los requisitos y formas de su organización".

${ }^{27}$ Ley Provincial 11.273/95, 28 de septiembre, de los productos fitosanitarios. Establece las distancias para las fumigaciones tanto aéreas como terrestres en articulación con la clasificación toxicológica de sustancias (arts. 33 y 34$)$

${ }^{28}$ En este sentido afirma la Cámara de Apelaciones: "fácil resulta concluir que no contamos en relación a la toxicidad pregonada con una certeza científica absoluta. Ahora, tal ausencia ¿constituye un óbice para la toma de decisión en el presente, si de lo que en verdad se trata es de evitar daños a la salud? La respuesta que a mi juicio se impone es negativa; y, por tanto, me apresuro a señalar que la aplicación del principio precautorio realizado por el juez a quo es correcta, ya que el mismo invita a actuar antes de que se obtenga la prueba del riesgo real, hipótesis que se encuentra receptada jurisprudencialmente con nuestro derecho como argumento central a los fines de reconocer pretensiones ambientales". Fragmento sentencia "Peralta..." Segunda Instancia.

${ }^{29}$ Lo realizado por parte de la Cámara se funda en la normativa de forma aplicable: "Pueden, también, para mejor proveer, ordenar que se practique cualquier diligencia que estimen conducente y que no sea prohibida por Derecho, y prescindir de la lista de peritos cuando fueren necesarios conocimientos especiales dentro de determinada profesión" (art. 20 Código Procesal Civil y Comercial de la Provincia de Santa Fe).
} 
provincial y el Ministerio de Salud, para que confeccione un estudio epidemiológico que permita comparar si existe una diferencia en términos de salud de la población antes y después del comienzo de la actividad de producción de soja en la zona identificada.

Recibida la información solicitada, se reenvía el caso ante la primera instancia que confirma su sentencia anterior. Esta decisión vuelve a ser objeto de recurso de apelación y la Cámara vuelve a confirmar la sentencia del Tribunal a quo, quedando prohibido, por tanto, realizar fumigaciones con glifosato en los predios indicados en la resolución.

Es interesante mencionar que, en el conjunto de resoluciones dictadas en este procedimiento judicial, se advierten algunos temas novedosos.

Por una parte, la Cámara intenta reconstruir la controversia científica haciendo alusión a los diferentes estudios que existen en el país sobre el tema y lo articula con los conocimientos de los profesionales de la salud. Se reconstruye la controversia científica mediante la inclusión, en el cuerpo de la sentencia, de un extenso listado de publicaciones sobre este tipo de productos y sustancias en general, algunas de las cuales se refieren específicamente al glifosato; a lo que se agrega una referencia expresa a los datos proporcionados por los profesionales de la salud, que implica una novedosa apertura hacia áreas del conocimiento no institucionalizado, que son tomadas en consideración e influyen en el momento de definir decisiones judiciales. A su vez, y esto constituye un dato también interesante, se recogen los argumentos de quienes claramente plantean la inocuidad del uso de este tipo de productos cuando se siguen 'buenas prácticas'. Si retomamos la mencionada idea de "parlamento científico", podría afirmarse que aquí encontramos un buen ejemplo de ello, dado que se intenta analizar el estado de la cuestión desde el ámbito científico. Por último, a ello se suma una referencia expresa sobre la importancia de tener en cuenta y valorar la voz de los afectados ${ }^{30}$.

\footnotetext{
${ }^{30}$ En la Cámara esta cuestión de las vivencias personales también se introduce, "Además, y particularmente en casos controvertidos científicamente, se torna muy relevante considerar las "historias de vida", las "experiencias", los "saberes y conocimientos" de quienes conviven cotidianamente expuestos al riesgo de que se trate, en este caso los agroquímicos. Es necesario revalorizar el sentido común debido a que la ciencia no puede responder a todos los interrogantes. Se han realizado recientemente algunas publicaciones que recuperan y presentan historias de personas expuestas a los agroquímicos. Entre ellas se destaca: "Pueblos fumigados. Los efectos de los plaguicidas en las regiones sojeras" de Jorge Rulli "Daños colaterales. Las víctimas ocultas de la fumigación” del CEPRONAT". Fragmento “Peralta...”. Segunda instancia. Primera sentencia.
} 
Por otra parte, se utiliza el principio de precaución a los efectos de profundizar en el conocimiento requerido para decidir sobre la problemática llevada ante la jurisdicción. Y ello se articula con el conocimiento de otras instituciones, tanto universitarias como de la administración provincial. Estos informes, en particular el de la Universidad, son medulares para la confirmación de la sentencia. De hecho, en la segunda sentencia del Tribunal a quo, se retoman algunos párrafos, entre los cuales destaca la conclusión general que advierte la inexistencia de agroquímicos inocuos, a lo que sigue un análisis sobre el informe del Ministerio de Salud ${ }^{31}$.

Tal praxis es novedosa en la jurisprudencia argentina sobre riesgos ambientales y relativos a la salud humana en general - y a agrotóxicos en particular -, debido a que no sólo se advierte un esfuerzo por reconstruir el estado de la controversia científica sino que se convoca a instituciones a los efectos de conocer mejor el grado de conocimiento del que se dispone. Una vez obtenida esa información, se vuelve a decidir sobre el caso, cuestión que no deja de poner de relieve también la relación entre el discurso experto y la decisión judicial $^{32}$.

Casos de este tipo se han repetido y llevado ante los Tribunales en los últimos años. Recientemente una situación similar, en la Provincia de Buenos Aires, condujo también a la presentación de una acción de amparo para lograr la prohibición de fumigaciones terrestres que se estaban llevando a cabo a escasos metros de la vivienda de una familia en la localidad de Mercedes. En la provincia mencionada no existe previsión para las distancias sobre fumigaciones terrestres, por lo que se solicita que se aplique de manera analógica lo previsto para las fumigaciones por vía aérea ${ }^{33}$. El caso llegó hasta la Corte Suprema de

\footnotetext{
31 “a modo de hipótesis que explica los fenómenos observados, puede plantearse que la disminución de la prevalencia de consultas por los motivos potencialmente atribuibles a un "irritante externo" en el segundo cuatrimestre en comparación con el resto de los diagnósticos, tanto en adultos como en niños, puede asociarse a la ausencia de un "factor ambiental" en dicho período ... La hipótesis planteada se ve reforzada a partir de la información de fuentes primarias, especialmente la obtenida a partir de entrevistas a informantes clave ... A modo de cierre, puede decirse que si bien y a partir de los límites del diseño metodológico y de los recursos disponibles para desarrollar la investigación (recursos materiales y de tiempo), no se pudo concluir de modo irrefutable que la disminución de las consultas entre ambos períodos se deba a la prohibición de fumigar. Puede que sí, como que no. ... No obstante, la hipótesis planteada parece ser bastante plausible". Fragmento "Peralta..." Primera Instancia. Segunda sentencia.

${ }^{32}$ BOUTONNET, M. "La preuve du préjudice environnemental, entre science et droit" en Truilhé-Marengo, Eve (Org.) La preuve entre science et droit. Larcier. Paris. 2011.

${ }^{33}$ Decreto $\mathrm{N}^{\circ}$ 499/1991, 4 de marzo. Este decreto establece la prohibición de fumigaciones aéreas a 2 kilómetros de centros poblados (art. 38)
} 
Justicia de la Provincia, que en su sentencia Monsalvo, Cristina y otros c/ Delaunay, Jorge s/ amparo de agosto de 2012, revoca la resolución de la Cámara de Apelaciones que actuó como segunda instancia en la causa. En la resolución de la Cámara se había rechazado el amparo presentado por Monsalvo por considerar que no se cumplía con el requisito de inminencia del daño que presupone la acción de amparo. Y ello, debido a la existencia de posturas contradictorias sobre los efectos de los productos agrotóxicos en la salud y el ambiente. La Corte, en su resolución, efectúa un extenso análisis del principio de precaución, la doctrina que ha hecho referencia al mismo, así como la jurisprudencia que ya lo ha aplicado y lo aplica al caso ${ }^{34}$.

\section{La discusión sobre la constitucionalidad de normas locales que prohíben o limitan el uso de agrotóxicos}

El segundo problema que se ha llevado ante la instancia jurisdiccional es aquel que deviene del intento de atacar la constitucionalidad de normas locales tendentes a la prohibición del uso de este tipo de productos en su territorio. Existen, hasta la fecha, dos sentencias relevantes en este sentido. Una de ellas que apunta la inconstitucionalidad de la ordenanza local cuestionada y otra que, ante una norma de similares características, se pronuncia sobre su constitucionalidad.

La primera de las causas es "Chañar Bonito S.A. c/ Municipalidad de Mendiolaza s/amparo", con resolución firme de fecha 18 de septiembre de 2007 dictada por el Tribunal Superior de Justicia de la Provincia de Córdoba. En este caso, la empresa "Chañar Bonito" plantea una acción de amparo por considerar sus intereses vulnerados ante la sanción de la Ordenanza $n^{\circ} 390$ de 2004 de la localidad de Mendiolaza, que prohíbe no sólo el uso sino el tránsito, comercialización, distribución, fabricación, etc., de productos agrotóxicos en su territorio.

\footnotetext{
34 “Concluyo pues que en el sub discussio, los extremos fácticos acreditados vistos a la luz de las consideraciones jurídicas que anteceden tornan desacertadas las conclusiones del fallo en orden a la ausencia del recaudo de admisibilidad analizado, toda vez que dichos elementos permiten inferir una probabilidad cierta de que se verifique la consecuencia lesiva que se pretende evitar con la acción intentada. Para utilizar el giro gramatical vertido en la pieza recursiva: "... no está acreditada la absoluta inocuidad de los agroquímicos que el demandado utiliza a gran escala a escasos metros de la vivienda de los actores y con ello no se puede concluir que no exista -a la luz del principio precautorio- situación de peligro a la salud...". Fragmento “Monsalvo...".
} 
La Corte revoca la sentencia de la Cámara que había rechazado el amparo ${ }^{35}$. En la tercera instancia se da lugar a la acción, declarándose la inconstitucionalidad de la norma municipal. La línea argumental del Tribunal se centra en el tema competencial por encima de cualquier otra vertiente de la problemática. Es la potestad del municipio lo que se discute, sin aludir ni al derecho ambiental vigente ni a las consecuencias que para la salud y el ambiente existan respecto del uso de este tipo de productos. Así, la construcción argumental del Supremo Tribunal conduce a concluir que "La simple confrontación entre el marco jurídico reseñado y la ordenanza 390 del año 2004 dictada por la municipalidad de Mendiolaza patentiza la antijuridicidad de esta última, ${ }^{26}$. Se considera que la comercialización de este tipo de productos es de competencia nacional y no puede ser alterada ni disminuida localmente. Ahora bien, entre los argumentos desplegados sobre la jerarquía de las normas y la competencia de cada nivel estatal - nacional, provincial y local -, existe uno de relevante importancia. Afirma la Corte que "si bien la materia regulada atañe a las potestades de regulación y fiscalización propias del ejercicio del poder de policía de la comuna en materia de salubridad, dicho ejercicio debe subordinarse al régimen jurídico vigente en el Estado federal, al cual no puede desconocer sin un tinte científico, técnico o local que justifique tal proceder toda vez que es claro que la temática de los compuestos químicos de aplicación a la producción agropecuaria desborda los intereses locales de los municipios al involucrar cuestiones que interesan a la Nación toda, circunstancia en mérito a la cual se le ha conferido al gobierno federal el establecimiento de sus bases" ${ }^{37}$.

Si se sigue este razonamiento, el régimen municipal podría regular sobre el tema siempre que se encuentre dentro del marco dispuesto por la estructura federal de distribución de competencias. Ello así se determina en materia de salubridad pública en Argentina, lo que

\footnotetext{
${ }^{35}$ La Cámara había sostenido que ": (i) que la normativa local forma parte de una construcción constitucional ya que dota de contenido la distribución competencial que prevé el art. $41 \mathrm{CN}$ en materia de legislación de presupuestos mínimos sin excederse del ámbito de la autonomía municipal conforme lo estipulado no sólo en la Carta Magna nacional sino, también, en la Constitución de la Provincia de Córdoba, (ii) que no se cumple con el requisito de daño actual o inminente, "no ha sufrido el grave daño que sostiene el amparista, al haberse permitido por vía de cautelar la fumigación requerida con carácter urgente y nada le impide hacia el futuro dedicar su propiedad a otro tipo de explotación que no requiera el uso de agroquímicos, cumpliéndose de esa manera lo preceptuado por el Art. 1 de la norma en cuanto pretende estimular la producción ecológicamente sustentable".

${ }^{36}$ Ibídem.

${ }^{37}$ Ibídem.
} 
permitiría una apertura hacia este tipo de problemas. Ahora bien, dentro del párrafo transcrito se advierte un punto que es cardinal no soslayar. El tribunal hace referencia a la existencia de razones 'científicas', 'técnicas' o 'locales' que, de existir, podrían justificar medidas como las dispuestas por la ordenanza cuestionada. En el argumento del tribunal se plantea que el problema de los compuestos químicos desborda los intereses locales. Ahora bien, lo que no se ha considerado a lo largo de la argumentación de la Corte, es la existencia de una importante serie de investigaciones, datos e informes que advierten sobre la existencia de riesgos tanto para el medio ambiente como para la salud, derivados del uso de este tipo de productos. Varios interrogantes quedan planteados a partir de esta resolución. Por un lado, la cuestión de la distribución de competencias para la gestión de riesgos, lo que permite a la vez insistir en el problema de cual es el mejor nivel territorial para la gestión de estos riesgos. Por el otro, también abre la puerta a la reflexión sobre en qué medida, lo controvertido o incierto - que en la sentencia aparece como "razones técnicas o científicas" - podría actuar como fundamento para la toma de decisiones de tipo restrictivo como en este caso y cómo articularlo con el criterio de proporcionalidad.

El segundo asunto que resuelve sobre la constitucionalidad de una ordenanza local es "Speedagro SRL c/ Comuna de Arequito s/ recurso contencioso-administrativo" y ha sido sentenciado por la Sala II de la Cámara en lo Contencioso Administrativo de Rosario el 3 de octubre de 2011. En este caso, es la empresa "Speedagro" la que solicita se declare la inconstitucionalidad de los arts. 7 y 9 de la Ordenanza $N^{\circ} 965 / 11$ de la Comuna de Arequito. En esta norma se dispuso la prohibición total de uso de productos fitosanitarios de banda roja así como la utilización de algunos coadyuvantes ${ }^{38}$.

En la sentencia se efectúa una remisión a la causa "Chañar", y se señala una distinción en términos de "proporcionalidad de la medida", elemento medular del principio de precaución. Sostiene la Cámara que "la prohibición establecida en la Ordenanza de Arequito es acotada o circunscripta. El presente, diverge pues de algunos otros casos que han sido fallados en la Argentina en los que los municipios y comunas habían prohibido la

\footnotetext{
${ }^{38}$ SENASA efectúa la clasificación de los productos fitosanitarios según su nivel de toxicidad, los productos en 'banda roja' son considerados como 'extremadamente tóxicos'. En la Ordenanza mencionada se estableció: Art. 7: Prohíbese el uso de productos fitosanitarios tóxicos y muy tóxicos (color de banda roja clase $1^{\mathrm{a}}$ y $1 \mathrm{~b}$ ) en todo el distrito Arequito: Metamidofos, lambdacialotrina al 25\%. Art. 9: Prohíbese el uso de coadyuvantes a base de nonilfenol etoxilados aplicados al tanque del equipo de aplicación en todo el distrito de Arequito por tratarse de sustancias capaces de alterar el sistema hormonal.
} 
utilización de cualquier tipo de producto químico o biológico de uso agropecuario, como es el caso de la Municipalidad de Mendiolaza (Provincia de Córdoba), prohibición tan radical que podría suscitar unos reproches desde la perspectiva de ejercicio del derecho al trabajo, a las actividades lícitas y al ejercicio del comercio" 39 .

Luego se realiza una extensa referencia al principio precautorio, incluso en el derecho comparado, y se sostiene que la controversia científica es esencial para fundamentar normas restrictivas como la atacada. Se concluye que “...Precisamente, la duda o incertidumbre científica torna aplicable el principio precautorio que autoriza a la Comuna a proceder como lo hizo... Justamente la "controversia" reconocida por la incidentista torna aplicable el precipuo principio que da pábulo a la posición de la administración comunal en este caso" ${ }^{40}$.

En las dos resoluciones estudiadas se aborda el tema de la competencia local para analizar la regulación de este tipo de problemas vinculados a riesgos ambientales y relativos a la salud. Ello es puesto en entredicho por parte de las empresas demandando la centralidad del Servicio Nacional de Sanidad y Calidad Agroalimentaria (SENASA), que es la agencia nacional competente, a través de su Dirección de Agroquímicos y Biológicos, en materia de elaboración, seguimiento, aplicación y supervisión de las normas y reglamentaciones sobre producción, comercialización y uso de productos agroquímicos y biológicos. Esta estrategia conduce a visualizar la cuestión de la concurrencia de competencias modalizada que propone la Constitución Nacional desde la reforma de 1994. Su artículo 41 establece que la Nación es la encargada de dictar las leyes que contengan los presupuestos mínimos de protección ambiental y las Provincias aquellas que fueran necesarias para complementarlas, sin que ello implique alterar las jurisdicciones locales. La cuestión, entonces, consiste en dilucidar cuáles serían esas competencias locales en torno a los temas que nos ocupan, que representan hipótesis de riesgos controvertidos que pueden afectar el ambiente y/o la salud. Algunas respuestas se encuentran en la última resolución analizada, en la que se establece que "en principio, no podrían soslayarse las competencias y potestades municipales y comunales en materia de salubridad (art. 45 inc. $1^{\circ}$ de la ley 2439 y art. 39 inc. 62 de la ley 2756) y en materia ambiental. La materia regulada en la Ordenanza se vincularía con

\footnotetext{
${ }^{39}$ Sentencia "Speedagro..."

${ }^{40}$ Ibídem.
} 
potestades de regulación y fiscalización, en principio, propias del ejercicio del poder de policía de la Comuna en las precipuas materias...En el caso, no puede soslayarse que estaríamos en presencia de una gestión concurrente de intereses comunes, pero en jurisdicción propia, fundada en poderes propios de la Comuna de Arequito".

\section{LA CONSTRUCCIÓN DE ESPACIOS Y MECANISMOS PARA LA DECISIÓN SOBRE RIESGOS}

Al margen de heterogéneos procesos de resistencia en relación al problema de las fumigaciones, además de las reclamaciones que comienzan a proliferar ante la instancia judicial, se advierten incipientes demandas sobre la necesidad de diseñar e implementar espacios institucionales y procedimientos apropiados para la toma de decisiones ante hipótesis precautorias. Ello no es ajeno al problema del uso de agrotóxicos. De hecho, este caso permite visualizar cómo colisiona una racionalidad preventiva para el gobierno del riesgo, que subyace a los espacios que definen diferentes prismas del tema, con situaciones en las que lo medular es la incerteza o controversia científica y no la posibilidad de prever consecuencias. Se colocan en tela de juicio las construcciones preventivas para la gestión de riesgos así como el ámbito científico como espacio que monopoliza el saber. Existen conocimientos que circulan más allá de este último, cuestionando la racionalidad científica moderna, como se ha señalado ya desde el campo tanto de la sociología del riesgo como de los estudios sociales de la ciencia. Las diferentes miradas en pugna ofrecen un escenario mucho más complejo que la sola controversia en el seno del campo científico. Y, llevado al plano de los espacios institucionales y los procedimientos para el gobierno del riesgo, introduce interrogantes y desafíos medulares a la hora de responder sobre quienes poseen legitimidad para participar en la toma de decisiones, o mejor, en la construcción de decisiones colectivas sobre riesgos colectivos.

\section{Diseños institucionales para la evaluación de riesgos}

Argentina no cuenta con un sistema jurídico/institucional para la gestión de riesgos científico-tecnológicos en general. Por el contrario, existen normas sectoriales que son las que se ocupan de forma parcial de los heterogéneos temas relacionados con la hipótesis de riesgos. En el tema de agrotóxicos se disponen dos niveles. Por una parte, el nacional, que a 
través de la Dirección de Agroquímicos y Biológicos autoriza la circulación, producción, etc., de este tipo de productos como se señaló en el apartado II.2. El trámite de autorización se configura mediante los datos que presenta el proponente y, luego, dentro de este organismo competente, se realiza el control del cumplimiento de las normas técnico/administrativas seguidas en la elaboración del fitosanitario y la evaluación técnica de la documentación presentada lo que se contradice con estrategias más corporativistas de evaluación de riesgos ${ }^{41}$. Por la otra, el nivel local, que determina las modalidades de aplicación en el territorio. Es cada Provincia la que regula los criterios a través de normas relativas al uso de este tipo de productos conforme se realice la fumigación de manera aérea o terrestre y según el tipo de sustancia de que se trate. En este esquema se verifica una perspectiva más vinculada con una estrategia preventiva que precautoria, lo cual comienza a ser analizado por diferentes actores sociales involucrados en este problema. La demanda por mejores espacios para la toma de decisiones ante hipótesis de precaución, empieza a aparecer pese a ser este principio uno de los pilares de la política ambiental desde hace ya más de una década. En Argentina, sin embargo, el principio precautorio ha tenido mayor trayectoria a nivel judicial que como inspiración de procedimientos y espacios institucionales para la toma de decisiones sobre riesgos de este tenor. Sin perjuicio de ello, se podría visualizar el inicio de algunas incipientes estrategias que podrían ser presentadas como de inspiración precautoria.

Con respecto al riesgo asociado a la dispersión de agrotóxicos, se ha creado recientemente una estructura que trabaja en la tarea de conocer de manera más profunda los posibles efectos de los agro-tóxicos sobre la salud, poniendo el acento así en la cuestión de la evaluación de riesgos. Se trata de la Comisión Nacional de Investigación sobre Agroquímicos, creada por Decreto $N^{\circ}$ 21/2009 del Ministerio de Salud de la Nación, una comisión de asesoramiento que se encuentra dentro de la órbita del Ministerio de Salud y está integrada por representantes de la Secretaría de Ambiente y Desarrollo Sustentable, la Secretaría de Agricultura Ganadería y Pesca, el Instituto Nacional de Tecnología

\footnotetext{
${ }^{41}$ Sobre la distinción entre dos tipos de políticas de evaluación de riesgo que puntualizan sobre (i) autorización vía decisión política fundada en dictámenes de grupos técnicos independientes o (ii) sistemas más corporativistas basados en la evaluaciones realizadas por los mismos industriales consultar HERMITTE, M. A. y DAVID, V. "Avaliação dos riscos e princípio da precaução" en Dias Varella, M.y Barros Platiau, A. F. Principio da precaução Coleção Direito Ambiental em Debate. Del Rey. Belo Horizonte. 2004.
} 
Agropecuaria, el Instituto de Tecnología Industrial y la Administración Nacional de Medicamentos, Alimentos y Tecnología Médica. Su misión consiste en la “...investigación, prevención, asistencia y tratamiento en casos de intoxicación o que afecten, de algún modo, la salud de la población y el ambiente, con productos agroquímicos en todo el territorio nacional..." (art. 1 Decreto N²1/2009 Min. Salud).

La mencionada Comisión, si bien ha sido creada para atender, preeminentemente, al tema de la evaluación de riesgos, además trabaja sobre cuestiones relativas a su gobierno y a la generación de medidas para mejorar el manejo de los agroquímicos ${ }^{42}$.

Sobre el problema de la evaluación, es importante efectuar algunas observaciones. En primer lugar, cabe indagar sobre la efectiva existencia de un espacio transdisciplinario en el seno de esta Comisión o, por el contrario, si éste se encontraría monopolizado por conocimientos vinculados con lo que se suele etiquetar como 'ciencias duras' en detrimento de otro tipo de saberes. Este tipo de pautas en relación a la transdisciplina, son sumamente necesarias en el abordaje de hipótesis de precaución que, justamente, se caracteriza por la existencia de una controversia científica que da lugar a posiciones divergentes y minoritarias. Esto se advierte como una tarea sumamente relevante en el diseño que asumen organizaciones de esta naturaleza en cuanto a su funcionamiento. Del mismo modo, podría continuarse el análisis respecto de los canales que se abren o no en el ámbito de otro tipo de saberes en respuesta al problema de la extensión y legitimidad para la toma de decisiones. Es decir, esbozar en cada caso concreto cuáles han de ser los requisitos necesarios para que se acrediten las opiniones del ámbito científico y de otros espacios que han producido un repertorio importante de datos e información sobre el tema, lo que es especialmente visible en el caso de los agro-tóxicos en Argentina. Por último, sería también esencial establecer

\footnotetext{
42 Ello se deduce de la constitución de los grupos de trabajo y del contenido de la norma que crea el organismo que establece como objetivos: "1. Investigar los hechos denunciados y situaciones similares, sus causas y efectos; 2. Efectuar recomendaciones, proponer acciones, planes, programas, etc.; 3. Delinear pautas para contribuir al uso racional de químicos y agroquímicos; 4. Proponer herramientas de información adecuadas para su utilización en los medios de comunicación; 5. Identificar los problemas generales en la atención sanitaria de la población afectada; 6. Desarrollar estrategias de atención para promover el uso racional de los productos o bien su eliminación; 7. Reunir información estadística e indicadores de impacto; 8. Propiciar la normativa pertinente y proponer las acciones directas a implementar; 9. Proponer campañas de concientización y educación sobre el uso, manipulación de químicos y agroquímicos; 10. Implementar, ejecutar y/o coordinar acciones, planes, proyectos y programas; 11. Realizar todas aquellas acciones que le encomiende la Presidencia de la Comisión" (art. 3 Decreto N²1/09 Min. Salud)
} 
cuáles son los criterios de excelencia e independencia requeridos para integrar este espacio $\mathrm{y} / \mathrm{u}$ otros espacios de esta raigambre.

\section{Procedimientos legales referidos a riesgos}

Como anteriormente se mencionó, existen normas locales o provinciales que determinan las condiciones de uso en el territorio de agrotóxicos, lo que se traduce en una serie de procedimientos previos de autorización y se articula con las pautas que rigen ambos temas en el derecho nacional. En general, en el tema de las fumigaciones con agrotóxicos, las normativas provinciales son las que pautan una cantidad de metros que operan a manera de límite de protección y que suelen diferir conforme la aplicación sea área o terrestre, para lo que debe presentarse ante la autoridad de aplicación una solicitud de autorización previa a la realización de la actividad. Es este el punto de disputa más relevante que se visibiliza en los intentos frustrados de reforma de la legislación vigente en diferentes provincias del país. Este tipo de normativa posee una relación inescindible con la regulación nacional. Se requiere la inscripción en el ámbito nacional del producto que se aplica, a lo que suelen agregarse, y esto sí ya constituye materia de competencia local, previsiones en torno a cuestiones más puntuales y específicas con respecto a su uso, como son la receta agronómica, el registro de aplicadores, el tratamiento de los envases, etcétera.

En casi la totalidad de las provincias se cuenta con una ley sobre el tema ${ }^{43}$. Este conjunto de legislaciones provinciales regulan el establecimiento de la autoridad de aplicación dentro del organigrama de las agencias estatales de cada provincia. Asimismo, se estipula un registro de productos, expendedores y aplicadores de agroquímicos así como de aplicadores. También se fijan las medidas de fiscalización y control y la regulación de

\footnotetext{
${ }^{43}$ En Buenos Aires la Ley N ${ }^{\circ}$ 10.699/1998 sobre agroquímicos, en Catamarca la Ley N ${ }^{\circ} 4395 / 1986$ sobre uso de productos agroquímicos, en Chaco Ley Nº7032/2012 de biocidas, en Chubut Ley N ${ }^{\circ} 4073 / 1995$ sobre regulación de las acciones relacionadas con biocidas y agroquímicos, en Córdoba Ley $N^{\circ}$ 9164/2005 de productos químicos o biológicos de uso agropecuario, en Corrientes Ley $\mathrm{N}^{\circ}$ 5300/1998 sobre régimen regulatorio del uso de agro-tóxicos, en Entre Ríos Ley $\mathrm{N}^{\circ}$ 6599/1980 de plaguicidas, en Formosa la Ley $\mathrm{N}^{\circ} 1163 / 1995$ de productos fitosanitarios, en Jujuy Ley $\mathrm{N}^{\circ} 4975 / 1996$ de sanidad vegetal, en La Pampa Ley $\mathrm{N}^{\circ} 1173 / 1988$ de agroquímicos, en La Rioja Ley $\mathrm{N}^{\circ}$ 9170/2011 de agroquímicos, en Mendoza Ley №5665/1991 de agroquímicos, en Misiones Ley $N^{\circ}$ 2980/1992 de uso de agro-tóxicos, en Neuquén Ley $\mathrm{N}^{\circ} 1859 / 1990$ de uso de biocidas, en Río Negro Ley $\mathrm{N}^{\circ}$ 2175/1987 de regulación de agroquímicos, en San Juan Ley $\mathrm{N}^{\circ} 6744 / 1996$ de fitosanitarios, en San Luis Ley $\mathrm{N}^{\circ} 5559 / 2004$ de regulación de uso de agroquímicos, en Santa Cruz Ley N ${ }^{\circ} 2484 / 1998$ y N²529/1999 de régimen de fitosanitarios, en Santiago del Estero Ley $\mathrm{N}^{\circ}$ 6312/1996 de agroquímicos, en Santa Fe Ley $\mathrm{N}^{\circ} 11.273 / 1995$ sobre uso de productos fitosanitarios, en Tucumán Ley $\mathrm{N}^{\circ} 6291 / 1991$ de agroquímicos.
} 
aspectos referidos al uso de los productos y sus condiciones de empleo, dentro de las cuales destacan las modalidades de aplicación de agroquímicos y las zonas en que se permite. Es decir, a cuantos metros del ejido urbano se pueden utilizar este tipo de productos en las fumigaciones, ya sea vía terrestre o área. Esta problemática concreta ha comenzado a ser objeto de debate y entre los argumentos utilizados, se encuentra el principio de precaución que fundamenta, por ejemplo, las propuestas de prohibición de la fumigación aérea y que, asimismo, se empieza a utilizar también en el debate suscitado en torno a la cantidad de metros desde los que puede o no fumigarse según el tipo de aplicación y de sustancia.

Se coloca, así, en un primer plano, la cuestión del alejamiento/proximidad al agente de riesgo lo que, además, suele vincularse con las clasificaciones toxicológicas de las sustancias. De una lectura de las normas provinciales que prevén esta cuestión, se extrae que existen dos elementos fundamentales para establecer las distancias. Por un lado, el tipo de fumigación aérea o terrestre y, por el otro, la categorización toxicológica del producto, a través de lo cual se establece un determinado uso que enlaza la previsión provincial con las potestades de los municipios en relación al tema. Ambos aspectos se encuentran cuestionados. El primero en función de los intentos por construir una nueva metodología clasificatoria de los niveles de toxicidad que considere los efectos a largo plazo y crónicos. A ello se suma la discusión cada vez más actual en torno a la determinación de las distancias en las que no puede llevarse a cabo la aplicación de estos productos por medio de fumigaciones, en las que surge con fuerza la propuesta de ampliar la cantidad de metros y se agrega la de prohibir la fumigación aérea con fundamento en el principio de precaución. Esta discusión en torno al uso en el territorio de productos agrotóxicos permite visualizar la controversia sobre la determinación de la cantidad de metros en los que puede o no fumigarse, lo que muestra el desafío en torno a evidenciar cómo se construyen tales criterios y quiénes son los actores sociales legitimados en tal debate. Discusión que también comienza a desarrollarse a nivel local, donde en los últimos años han proliferado una serie de normativas que intentan restringir el uso de este tipo de productos y que, paralelamente, son atacadas en cuanto a su constitucionalidad como se analizó en el apartado II.2. 


\section{ALGUNAS REFLEXIONES FINALES}

Este trabajo ha tenido por objeto visibilizar la aplicación del principio de precaución en la reciente jurisprudencia argentina, así como su incipiente presencia en las reclamaciones tendentes a conseguir una mejora en el diseño de instituciones y procedimientos para la toma de decisiones sobre esta hipótesis de precaución. Para ello, se seleccionó como caso puntual el uso de agrotóxicos, problema de especial actualidad y relevancia. En el plano jurisdiccional se identifican los problemas que han sido objeto de decisiones y que han proliferado en los últimos años, generando así un conjunto de resoluciones que aplican el principio precautorio en materia de dispersión de agrotóxicos. En el plano institucional y procedimental que permite la toma de decisiones sobre riesgos, se ha identificado, por un lado, la inexistencia de un sistema general de gestión de riesgos científico/tecnológicos. Por el otro, se ha observado un esquema más bien de naturaleza preventiva que subyace en el registro y clasificación de productos agrotóxicos y aplicación en el territorio y que actualmente comienza a encontrarse con reclamos por otro tipo de estructuras inspiradas en el principio precautorio. En ese sentido, se verifica una muy incipiente creación de un espacio para la profundización del conocimiento sobre el tema, lo que puede ser interpretado en clave precautoria, pero que requiere de una serie de mejoras relevantes como la necesidad de reforzar los criterios de excelencia en la elección de los expertos que la componen, su independencia, la necesidad ineludible de expresar las diferentes posiciones que se encuentran en el plano científico y, asimismo, la integración de otros saberes disponibles. En cuanto a los procedimientos de índole más local, se advierte la existencia de regulaciones que, fundamentadas en la precaución, limitan el uso de agrotóxicos, lo que pone de relieve la cuestión de cuál es el mejor nivel territorial para la gestión de riesgos y el desafío de la coordinación e integración de este tipo de estrategias de gobierno en los diferentes niveles territoriales.

Son numerosas las agendas planteadas, abiertas y por abrir, en un camino que parece comenzar con dificultades. Este tema permite realizar una reflexión más amplia en torno a qué puede hacer el derecho para contribuir al gobierno de riesgos controvertidos. 


\section{REFERENCIAS BIBLIOGRÁFICAS}

\section{Libros:}

BECK, U. La sociedad del riesgo. Hacia una nueva modernidad, Paidos, Barcelona, 1997.

BECK, U. La sociedad del riesgo global, Siglo XXI, Barcelona, 2002.

BECK, U. La sociedad del riesgo mundial, Paidos, Barcelona, 2007.

CASTEL, R. La montée des incertitudes. Travail, protection, statut de l'individu, Editions du Seuil, Paris, 2009.

DE SADELEER, N. Environmental principles. From political slogans to legal rules, Oxford University Press. Oxford. 2002.

DOUGLAS, M. y WILDAVSKY, A. Risk and culture. An essay on the selection of technological and environmental dangers, University of California Press. California. 1983.

EMBID TELlO, A. Precaución y derecho. El caso de los campos electromagnéticos, Isutel. Madrid, 2010.

ESTEVE PARDO, J. El desconcierto del Leviatán. Política y derecho ante las incertidumbres de la ciencia, Marcial Pons. Madrid, 2009.

ESTEVE PARDO, J. Técnica, riesgo y derecho. Tratamiento del riesgo tecnológico en el Derecho Ambiental, Ariel. Barcelona, 1999.

EWALD, F. Le principe de précaution, Que sais je ? PUF. Paris, 2001.

GIDDENS, A. (1993) Las consecuencias de la modernidad, Alianza. Madrid.1993

HERMITTE, M. A. “La fondation juridique d'une société des sciences et des techniques par les crises et les risques", Burton-Jeangros, C ; Grosse C. y November, V. (dir.) Face au risque, Georg-L'Equinoxe, 2007.

HERMITTE, M. A. "Los fundamentos jurídicos de la sociedad del riesgo. Un análisis de Ulrich Beck” Sozzo, G. (dir) El gobierno del riesgo Ediciones UNL. Santa Fe. 2007. 
HERMITTE, M. A. “La dissémination volontaire d'organismes génétiquement modifiés dans l'environnement une première application du principe de précaution" Revue Juridique de l'Environnement $\mathrm{N}^{\circ} 3 / 93$.

LATOUR, B. Politiques de la nature. Comment faire entrer les sciences en démocratie, La Découverte, Paris, 2004.

LATOUR, B. Nous n'avons jamais été modernes. Essai d'anthropologie symétrique La Decouverte. Paris, 1997. Versión en español LATOUR, B. Nunca fuimos modernos. Ensayo de antropología simétrica Siglo XX Editores. Buenos Aires, 2007.

LUPTON, D. Il rischio. Percezione, simboli, cultura Il Mulino, Universale Peperbacks. Bologna. 2003.

NOIVILLE, C. Du bon gouvernement des risques. Le droit et la question du "risque acceptable”, Presses Universitaires de France, Paris, 2003.

PESTRE, D. Introduction aux sciences studies, La Découverte. Paris. 2006.

SANTOS, B. Para descolonizar Occidente. Más allá del pensamiento abismal, CLACSO. Prometeo Libros. Buenos Aires, 2010

SOZZO, G. y BERROS, M. V. "Una agenda para el principio precautorio" Revista Crítica de Derecho Privado Nº -2009, La Ley, Uruguay.

\section{Capítulos de libros:}

HERMITTE, M. A. y DAVID, V. Avaliação dos riscos e princípio da precaução Dias Varella, M.y Barros Platiau, A. F. (Dir) Principio da precaução Coleção Direito Ambiental em Debate, Del Rey, Belo Horizonte, 2004.

NOIVILLE, C. "Science, décision, action: trois remarques à propos du principe de précaution" Petites affiches. 1er -2. N. 218-219, Paris, 2004.

\section{Artículos de revistas:}

BERROS, M. V. "La estandarización como tecnología precautoria globalizada. El caso de las radiaciones no ionizantes" Volumen 5, №2 Revista Prismas Direito, políticas públicas e mundializaçao. Colección Universitaria UNICEUB. Brasilia, 2008. 
BROWN, P. "Epidemiology and Toxic Waste Contamination: Lay and Professional Ways of Knowing" Journal of Health and Social Behavior, Vol. 33, No. 3 (Sep., 1992), pp. 2672.

MORALES LAMBERTI, A. "Conflictos de reglas, principios y paradigmas en la decisión de un caso ambiental complejo: agroquímicos y facultades locales" Revista de Derecho Ambiental, núm. 14, 2008. 